\title{
Model-Based Integration and Interpretation of Data
}

\author{
Petersen, Johannes
}

Published in:

IEEE International Conference on Systems, Man \& Cybernetics

Link to article, DOI:

10.1109/ICSMC.2004.1398403

Publication date:

2004

Document Version

Publisher's PDF, also known as Version of record

Link back to DTU Orbit

Citation (APA):

Petersen, J. (2004). Model-Based Integration and Interpretation of Data. In IEEE International Conference on Systems, Man \& Cybernetics (Vol. 1, pp. 815-820). IEEE. https://doi.org/10.1109/ICSMC.2004.1398403

\section{General rights}

Copyright and moral rights for the publications made accessible in the public portal are retained by the authors and/or other copyright owners and it is a condition of accessing publications that users recognise and abide by the legal requirements associated with these rights.

- Users may download and print one copy of any publication from the public portal for the purpose of private study or research.

- You may not further distribute the material or use it for any profit-making activity or commercial gain

- You may freely distribute the URL identifying the publication in the public portal

If you believe that this document breaches copyright please contact us providing details, and we will remove access to the work immediately and investigate your claim. 


\title{
Model-Based Integration and Interpretation of Data ${ }^{*}$
}

\author{
Johannes Petersen \\ Ørsted•DTU, Automation \\ Technical University of Denmark \\ jop@oersted.dtu.dk
}

\begin{abstract}
Data integration and interpretation plays a crucial role in supervisory control. The paper defines a set of generic inference steps for the data integration and interpretation process based on a three layer model of system representations. The three layer model is used to clarify the combination of constraint and object-centered representations of the work domain throwing new light on the basic principles underlying the data integration and interpretation process of Rasmussen's abstraction hierarchy as well as other model-based approaches combining constraint and object-centered representations. Based on these results some implications for interface design are outlined.
\end{abstract}

Keywords: Cognitive Systems Engineering, work domain analysis, data integration, data interpretation, abstraction hierarchy, constraints.

\section{Introduction}

In Cognitive Systems Engineering, representations of the work domain, i.e. the system with which operators are supposed to interact through the interface, has been a major focus area. It has been argued that representations based on constraints, specifying proper functioning, are fundamental in order to cope with unanticipated events (e.g. [5], [20]).

A primary task in supervisory control is to identify the state of the work domain based on measurements of key variables. This task comprises integration, derivation and interpretation of data and requires a viable representation of the work domain. The representation is supposed to serve both as an interpretative framework, enabling identification and interpretation of significant events (deviations from proper functioning), and as a reasoning framework enabling causal reasoning about the relations between events.

The paper describes the data integration and interpretation process using a model of the structural relations among three different forms of system representation that typically take part in work domain representations:

1. A representation given in terms of causal relations among objects.

2. A representation given in terms of constraints (relations between variables).
3. A representation given in terms of variables.

The model is referred to as the three layer model (TLM) and is used to define a set of generic inference steps in the data integration and interpretation process. These inference steps involve data at all three layers of system representation. At the variable layer data reflect the quantitative and qualitative value of variables, at the constraint layer data reflect the state of constraints, and at the object-centered layer data reflect the state of objects.

The paper discusses a combination of the three layer model and Rasmussen's abstraction hierarchy [16]. The abstraction hierarchy and the three layer model are orthogonal. The former focuses on the relevant content of the work domain (given at different means-end abstraction levels), whereas the latter focus on the relevant form of such representations.

The combination of the abstraction hierarchy and the three layer model leads to a clarification of some fundamental unresolved issues related to the structure and form of work domain representations based on the abstraction hierarchy as well as its use in the data integration and interpretation process. More specifically, the three layer model makes explicit how constraint and object-centered representations of the work domain can be combined in a systematic manner. Something that is absolutely fundamental in order to support the data integration and interpretation process.

The proposed inference steps defined on the basis of the three layer model throw new light on the basic principles underlying the data integration and interpretation process of the abstraction hierarchy as well as other model-based approaches combining constraint and object-centered representations, such as e.g. Multilevel Flow Modeling [7], [8], [9]. In other words, it is explained how such representations can serve both as a framework for interpretation and reasoning.

Based on these results some implications for interface design are outlined.

\section{The three layer model}

The three layer model makes a distinction between three layers of system representation (variables, constraints, and objects and relations) (see Fig. 1).

* 0-7803-8566-7/04/\$20.00 @ 2004 IEEE. 


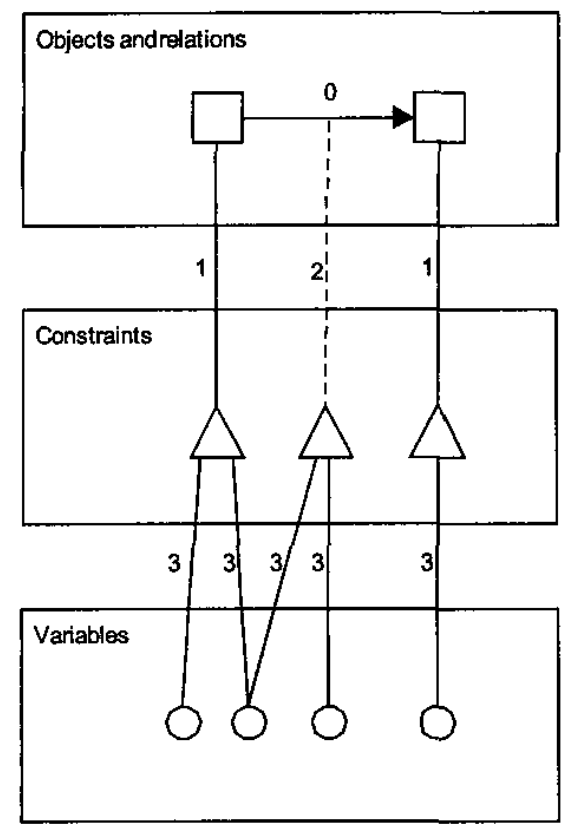

Fig. 1. The three layer model of representational forms.

The upper layer contains a representation of the work domain in terms of objects that interact causally. A causal relation among two objects exists if a state change in the first object can produce a state change in the second object (see the type 0 relation in Fig. 1). The actual symbolic reference of the objects and relations depends on the conceptualization of the work domain. According to Rasmussen [16] a work domain can be conceived at different levels of abstraction along the means-end dimension. In principle, the objects in the three layer model may refer to any of these levels, but here it is presumed that objects refer to functions at a specific level of means-end abstraction'.

For an example of a formalized method for causal reasoning based on an abstract function-oriented representation see [11] and [12].

Representations based on relations among objects are reflected in natural language and form the basis for commonsense causal reasoning (see e.g. [15], [16], [18], [19]). This feature of object-centered representations makes them highly relevant in a human-machine context.

The next layer in the three layer model contains a set of constraints describing relations between variables or relations between variables and constants. Typically, constraints are formulated in terms of mathematical relations between variables but may e.g. also be described by a graph in a coordinate system. Some of the constraints

\footnotetext{
${ }^{1}$ Note that although the means-end dimension is left implicit in the three layer model, it is actually necessary to refer to specific ends when formulating the functions at the object-centered layer.
}

specify the proper function of the functions at the objectcentered layer, enabling an identification of the state of the functions. Other constraints may be used to specify causal relations among objects. However, as argued below this requires a causal interpretation of the constraints.

Finally, the bottom layer of the three layer model contains system variables of which only a subset enters into constraints.

\subsection{Types of representation}

As argued by Rasmussen [16] constraint representations and object-centered representations, reflected in the three layer model, give rise to complementary representations of the work domain. "The two types of descriptions are in many respects complementary. In causal models, objects interact by events; the system is a set of objects related by a net of potential interactions in which changes or events propagate. Several quantitative variables are typically necessary to replace a description in terms of a state of a component or a mutual event between two components. The formal deterministic model is a network of relations among variables. In this model, the variables have replaced the physical objects as elements of the model. Physical objects are dissolved into a set of relations." [16, p.145].

The three layer model attempts to make explicit how these complementary types of representations can be combined in work domain representations. In the following section the structural relationships among the different layers are described in greater detail.

\subsection{Structural relations}

Between the object-centered layer and the constraint layer there are relations describing links between objects and constraints specifying proper functioning (type 1 relations in Fig. 1). These relations are many-to-many relations because several constraints may be needed to specify the proper functioning of an object-centered description of a function and because the same constraints may be used to specify the proper functioning of several functions.

What about the causal relations among objects, can they also be specified by constraints at the constraint level? The first answer to this question is no. Mathematical functions or constraints express constant relations among the numerical values of (metrical) properties and can be used to state that something is invariably associated with something else. Mathematical functions make it possible to symbolize and to give precise quantitative descriptions and predictions of connections but they fail to state the one-sided (asymmetric) connection (causal production) that characterizes causation [2].

This does not mean, however, that constraint descriptions cannot be used to infer information about causal relations at the object-centered layer. In order to do this it is necessary to perform an interpretation of the 
mathematical function (constraint). Bunge [2] argues that: "[Mathematical] functions, together with semantic rules stating the meaning of the variables tied by them, are often useful to tell what happens and why it happens; if a causal meaning can be attached to some of the symbols intervening in a [mathematical] function (rarely, if ever, to all of them), such an interpreted [mathematical] function will reflect a causal connection. In other words, [mathematical] functions, which are syntactic forms, cannot replace causal propositions; at most, they may take part in the description of causal connections." [2, p. 95, emphasis in original].

Based on Qualitative Process Theory [3] such interpretations of a constraint could be expressed by socalled qualitative proportionalities between the state variables of the objects in question ${ }^{2}$. In the three layer model a specific type of relation is used to link a causal relation at the object level and the constraints that take part in its description. This relation type is indicated by a dashed line (type 2 relation in Fig. 1).

Finally, between the constraint layer and the variable layer, relations describe the link between constraints and the variables that enter into the constraints (type 3 relations in Fig. 1). Obviously, these relations are also many-to-many relation. Note that not all the variables need to enter into constraints.

\subsection{Example}

In order to illustrate the application of the three layer model the simplified reservoir system, shown in Fig. 2, is used as an example.

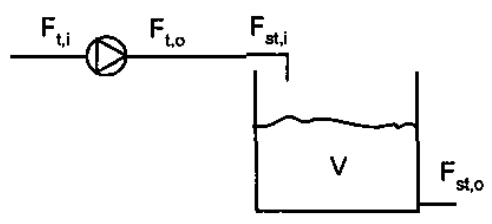

Fig. 2. The simplified reservoir system.

At the object-centered layer two objects represent the reservoir system (see Fig. 3). These objects denote a transport function and a storage function of the reservoir system, respectively. Each of the functions has a so-called state variable. The state variable for the transport function is the outlet flow $\left(F_{t_{0}}\right)$ whereas the state variable for the storage function is the volume of the reservoir (V).

${ }^{2}$ A variable V1 is qualitative proportional to another variable V2 if there is a mathematical functional relationship between $\mathrm{V} 1$ and $\mathrm{V} 2$, and $\mathrm{V} 1$ is increasing monotonic in its dependence on V2. Inverse qualitative proportionalities are defined similarly, with the function being decreasing monotonic.
The functions are related to constraints specifying proper functioning. The constraint specifying proper functioning of the transport (constraint number 1) expresses that the inlet flow $\left(\mathrm{F}_{\mathrm{t}, \mathrm{i}}\right)$ is equal to the outflow $\left(F_{t o}\right)$. For the storage there are two constraints ( 2 and 3 ). Constraint number 2 expresses that the rate of change of the volume $(\mathrm{dV} / \mathrm{dt})$ is proportional to the difference between the inlet flow $\left(\mathrm{F}_{\mathrm{st}} \mathrm{i}\right)$ and the outlet flow $\left(\mathrm{F}_{\mathrm{st}, \mathrm{O}}\right)$ of the reservoir. Constraint number 3 expresses that the volume $\mathrm{V}$ is supposed to stay below a certain limit $\left(\mathrm{V}_{\max }\right)$ in order for the storage to function properly.

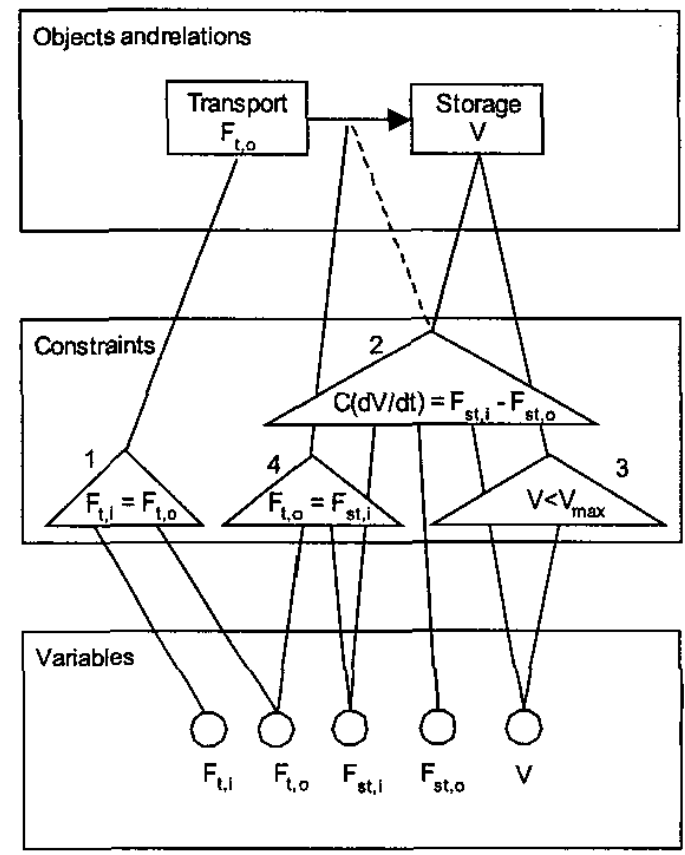

Fig. 3. A three layer for the reservoir system.

Fig. 3 shows that the transport function interacts causally with the storage function. The causal relation is derived by a causal interpretation of the conservation law specifying the proper function of the storage. The causal interpretation of this constraint expresses that the rate of change of the reservoir volume is proportional to the inlet flow of the reservoir. This means that a state change of the transport function may cause a state change in the storage function (presuming that the inlet flow of the reservoir is equal to the outlet flow of the transport as stated in constraint number 4). There is no causal relation from the storage to the transport because changes in the volume of the reservoir, will not influence the inlet flow of the reservoir.

\subsection{TLM and the abstraction hierarchy}

Rasmussen's abstraction hierarchy [16] specifies five levels of abstraction of a work domain organized along a means-end dimension. The levels of abstraction reflect 
different conceptualizations of the work domain that can be decomposed into parts along the part-whole dimension.

The bottom level of the hierarchy contains a representation of the physical components of the work domain. When moving upwards in the hierarchy, information about the physical implementation is discarded and information about the intended co-function of the components is added. At the top level, the purpose of the system is represented.

The abstraction hierarchy focus on the content of a work domain representation and is in fact orthogonal to the three layers of system description discussed above which focus on the form of work domain representations. For this reason it is fruitful to consider a combination of the three layer model and the abstraction hierarchy. One way of thinking about this combination is to have instances of the three layers of system representation at each level of abstraction. The symbolic reference of the objects and relations is determined by the conceptualization of the work domain at the given level of abstraction.

The means-end relation in the abstraction hierarchy link two objects at adjacent levels of abstraction. Seen from the point of view of the three layer model this type of relation corresponds to a relation among two objects that are part of parallel instantiations of the three layer model. The nature of the means-end relations among objects at different levels of means-end abstraction is virtually similar to the causal relation among objects at the same level (discussed earlier). This means that means-end relations may also be specified by a causal interpretation of constraints at the constraint layer.

In the literature it is hard to find discussions of different forms of representations of the work domain, let alone discussions of the relation between the three different layers of system representation discussed here. Sometimes the focus is on constraints: "Because the system was built in a certain way, for a certain purpose, there will be certain relationships between variables. These relationships can be described as constraints.... [the abstraction hierarchy] provides a framework for identifying and integrating the set of goal relevant constraints that are operating in a given work domain. Each level in the hierarchy represents a different class of constraints..." [20, p. 592] and sometimes the focus is on relations among objects: "The AH was used as a basis for developing a formal representation of DURESS. DURESS was described in terms of objects which comprise the system at each level of abstraction, along with the means-end links connecting those objects across levels" [1, p. 88].

In the literature describing applications of the abstraction hierarchy one typically finds separate representations of the variables, constraints and the relations among objects (e.g. [1], [4]). Exceptions to this rule can be found in [17], [10], but still the combination of constraint and objectcentered representation is not treated systematically.

Vicente [21] comments on the relation between an object-centered work domain analysis of DURESS II based on the abstraction hierarchy and the equations (constraints) governing the system, but refrains from a discussion of the link between the different forms of representation: "Although it may be difficult to believe, the work domain representation just described is actually a qualitative, function-oriented representation of the same relations that where identified in a quantitative, functionindependent form by the equations in Table $6.6 \ldots$ The structural means-end and part-whole relationships identified by the work domain analysis are actually implicitly represented in these equations goveming DURESS II." [21, p.177].

\section{Generic inference steps}

Generic inference steps (information flow) in the data integration and interpretation process, based on the three layer model of system representation, are illustrated in Fig. 4. Lind [6] has used a similar figure to illustrate the integration and interpretation process in MFM. The figure describes how the data values propagate between the different layers of system representation. At the variable layer data reflect the quantitative and qualitative value of variables, at the constraint layer data reflect the state of constraints, and at the object-centered layer data reflect the state of objects.

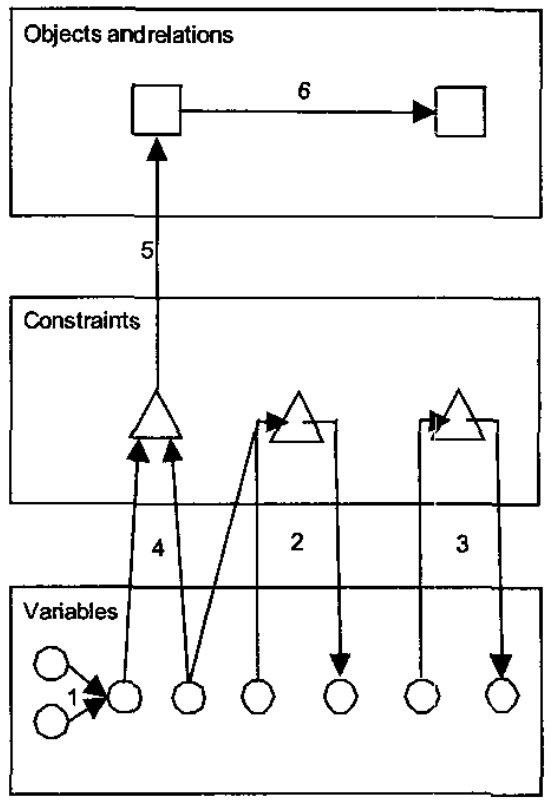

Fig. 4. The generic inference steps shown in the three layer model.

Below the different inference steps are described. The numbers in the text refers to the numbers in Fig. 4.

1) Integration. Integrating variables into higher-order variables.

2) Derivation. A constraint can be used to derive the value of a higher-order variable from the values of 
measured variables. The assumption here is that the constraint used is valid.

3) Variable state identification. A constraint of the interval type (e.g. defined by minimum and maximum values) can be used to identify the state (qualitative value) of a variable (low/normal/high or normal/abnormal).

4) Constraint state identification. Based on the value of the variables entering into a constraint, it is possible to identify the state of the constraints (violated/notviolated). When the values of the variable are inconsistent with the constraint defining the interrelations between the variables the constraint is violated. This, of course, relies on the assumption that the variable values are valid.

5) Object (function) state identification. Based on the state of constraints (violated/not-violated) it is possible to identify the state of objects (functions) at the object-centered layer. If the constraint(s) specifying the proper function is (are) violated the function is failed, otherwise the function is $o k$.

6) Causal reasoning based on an object-centered model. Reasoning about the state of the work domain based on an object-centered model representing causal relations among objects.

The role of constraints taking part in an interpretative framework has been emphasized in [11], [13], [17]. The inference steps just described reveal that the result of the comparison process between data and constraints can be ambiguous. For interval constraints, for example, the result of the comparison process can be viewed either as a classification of some variable or as an identification of the state of an object (function). In the former case, the quantitative value of a variable is transformed into a qualitative value of the same variable (e.g. low/normal/high or normal/abnormal). In the latter case the quantitative value of a variable is used to identify the state of a function (failed, ok) of the work domain. This distinction is described in detail in [14] using the notion of scale transformation.

In a human-machine context it is believed to be important to enable a judgment of the implication of a violated constraint at the object-centered layer comprising a representation of the goals and functions of the work domain. This puts strong demands on the interpretative framework being used, requiring an explicit representation of the relations between an object-centered representation and a constraint representation specifying proper functioning. Particularly, in unfamiliar situations it is crucial that operators know what a violated constraint actually means in terms of disturbed goals and functions at the object-centered layer. Only at this layer of system representation it is possible to reason about causes and consequences at a plant wide level.

\section{Implications for interface design}

A traditional one-sensor-one-indicator interface would simply show the values of the sensed and derived variables by means of separable dimensions. This approach to interface design provides poor support for the operator supervising a complex work domain because the operator is left with the burden of integrating and interpreting data.

A prototypical interface designed according to Ecological Interface Design principles (e.g. [20]) displays the variables that enter into the constraints of the work domain and allows the operator to compare perceptually, based on analytical geometry representing the constraints, the current behaviour of the work domain with the behaviour that is expected from the constraints.

A complementary approach to interface design is to make explicit the result of the comparison process, in terms of the state of objects referring to functions of the work domain. In this approach the constraints serve a secondary role. An appropriate visualization of the objectcentered layer (objects and their relations) would allow the operator to reason about the system at a plant wide level. Typically, this type of interface is referred to as an integral display.

\section{Conclusions}

A set of generic inference steps for the data integration and interpretation based on a model of the relation among three layers of system representation has been defined. The three layer model has been used to clarify how to combine constraint and object-centered representations of a work domain. This has thrown new light on the structure of work domain representation based on the abstraction hierarchy and the basic principles underlying the data integration and interpretation process of the abstraction hierarchy as well as other model-based approaches combining constraint representations and object-centered representations. It has been explained how such representations can serve both as a framework for interpretation and reasoning. Based on these results some implications for interface design has been outlined.

\section{References}

[1] A. M. Bisantz and K. J. Vicente, "Making the abstraction hierarchy concrete," Int. J. Human-Computer Studies, Vol. 40, pp. 83-117, 1994.

[2] M. Bunge, Causality. The place of the causal principle in modern science, Harvard University Press, Massachusetts, 1959.

[3] K. D. Forbus, "Qualitative Process Theory," Artificial Intelligence, Vol. 24, pp. 85-168, 1984. 
[4] D. H. Ham and W. C. Yoon, "Design of Information Content and Layout for Process Control Based on GoalsMeans Domain Analysis," Cognition, Technolog \& Work, vol. 3, pp. 205-223, 2001.

[5] M. Lind, "The use of flow models for automated plant diagnosis," in Human Detection and Diagnosis of System Failures, J. Rasmussen and W. Rouse, Ed. New York: Plenum, 1981.

[6] M. Lind, "Diagnosis Using Multilevel Flow ModelsDiagnostic Strategies for P96 Demonstrator" CEC ESPRIT project 96 - Expert System Builder, 1988.

[7] M. Lind, "Representing Goals and Functions of Complex Systems," Dept. of Automation, Technical University of Denmark, Tech. Rep. 90-D-381, 1990.

[8] M. Lind, "Modelling Goals and Functions of Complex Industrial Plants," Applied Artificial Intelligence, vol. 8, pp. 259-283, 1994.

[9] M. Lind, "Plant modelling for supervisory control," Trans Inst MC, Vol. 21. No. 4/5, pp. 171-180, 1999.

[10] Q. Liu, K. Nakata and K. Furuta, "Display Design of Process Systems Based on Functional Modelling," Cognition, Technolog \& Work, vol. 4, pp. 48-63, 2002.

[11] J. Petersen, "Knowledge Based Support for Human Supervisory Control," Ph.D. Dissertation, Dept. of Automation, Technical University of Denmark, Denmark, 2000.

[12] J. Petersen, "Causal Reasoning Based on MFM," in Proc. Conference on Cognitive System Engineering in Process Control (CSEPC), pp. 36-43, 2000.

[13] J. Petersen, "Situation Assessment of Complex Dynamic Systems Using MFM," in Proc. $8^{\text {th }}$ IFAC Symposium on Analysis, Design and Evaluation of Human-Machine Systems, pp. 645-650, 2001.

[14] J. Petersen and M. May, "Scale Transformations and Information Presentation in Supervisory Control," Int. Journal of Human-Computer Studies, submitted for publication.

[15] J. Rasmussen, "On the structure of knowledge - a morphology of mental models in a man-machine system context," Rep. RISø-M-2192, Risø Nat. Lab., Electronic Dep., Roskilde, Denmark, 1979.

[16] J. Rasmussen, Information Processing and HumanMachine Interaction. An Approach to Cognitive Engineering, Amsterdam, North-Holland, 1986.
[17] D.V.C. Reising and P. M. Sanderson, "Work domain analysis and sensors I: principles and simple example," Int. Journal of Human-Computer Studies, vol. 56, pp. 569-596, 2002.

[18] R. Schank and R. Abelson, Scripts plans goals and understanding, An inquiry into human knowledge structures, Hillsdale, NJ: Erlbaum, 1977.

[19] L. Talmy, "Force Dynamics in Language and Cognition," Cognitive Science, vol. 12:1, pp. 49-100, 1988.

[20] K.J. Vicente and J. Rasmussen, "Ecological Interface Design: Theoretical Foundations," IEEE Transactions on systems, man, and cybernetic, vol. 22, pp. 589-606, 1992.

[21] K.J. Vicente, Cognitive Work Analysis: Toward Safe, Productive, and Healthy Compuier-Based Work, Lawrence Erlbaum Associates, Publishers, Mahwah, 1999. 